\title{
Leadership selection when uncertainty is minimal'
}

\author{
ARNOLD BINDER, NEW YORK UNIVERSITY \\ BURTON R. WOLIN AND STANLEY J. TEREBINSKI, \\ SYSTEM DEVELOPMENT CORPORATION, SANTA MONICA, CALIFORNIA
}

\begin{abstract}
Methods used previously to test a Markov model for leadership selection in small groups were applied to a special set of reinforcement conditions for the decisions of the leader. In earlier runs, members were reinforced on a partial schedule, but in the present experiment one member was reinforced with probability 1 for his decisions. Partly, it appeared, because of an experimental artifact, fit for predictions from the model were obtained for only one of two sets of Ss.
\end{abstract}

\section{Introduetion}

Recent work by Binder, Wolin, \& Terebinski (1965 a, b, in press) has been directed at developing and testing a Markov model for a leader selection game in teams containing three members. The game consists of a sequence of trials, on each of which the members of a team vote for a leader and the designated leader then makes a decision for the team. The members of a team are not informed of the actual decision of the leader on trial, only whether his decision was right or wrong. This made it possible to control the reinforcement probability (\% right) for the decisions of each member when he acted as the team's leader.

The parameters in the mathematical model consisted of the reinforcement probabilities (fixed experimentally) of each member and the probability (estimated from data) of a shift in voting choice from trial $n$ to trial $\mathrm{n}+1$ for those contingencies where this probability was not assumed to be zero. Reinforcement probabilities were run in various combinations of the values $.9, .7, .5$, $.3, .1$; thus, for example, five teams were run in the combination .9 .5 .1 , meaning that one member in the team of three was informed that he was right for $90 \%$ of the decisions he made when leader, another was so reinforced $50 \%$ of the time, and the third $10 \%$. Despite certain weaknesses, the model provided generally good fits between obtained and expected results, especially over asymptotic trials. Matching and maximizing models were clearly inferior.

None of the reinforcement probability sets so far used included any probabilities above .9 . Since this is a particularly interesting set from the standpoint of placing a severe strain on the model as well as providing an experimental variant of general interest, an experiment was run to fill this gap.

\section{Method}

Six groups (teams), three Ss per group, were run for a total of 480 trials. One member in each group was reinforced with a probability $(\pi)$ of 1.0 , another at .5 , and the third at .3. Each trial contained a voting phase and a decision phase; all members voted but only the leader decided. The Ss were instructed to vote for either one of the other two members in their groups during the former phase and, when designated group leader, to indicate which of two alternatives was the correct decision during the latter phase. Responses in both cases were made by pushing buttons on a console.

All stimulus information was displayed on a cathoderay scope activated by an on-line computer. In addition to specification of the results of voting and decision processes and instructional statements appropriate to the phase, the scope contained a running score for each team (labeled "Group \% Right"). The score was provided to augment the preliminary instructions; these instructions attempted to motivate the Ss by specifying that the goal for a team was to get a higher score than any other team. Whenever a member failed to vote within the allowed time he became ineligible for the leadership role on that trial, and when a leader failed to decide within the allowed time his decision was designated as "wrong。" When there were two or more eligible members and none received a majority of the votes cast, the computer chose the leader randomly. The experimental sessions were arranged in such a manner that the members did not know the identities of their team-mates. The full run of 480 trials took about $2-1 / 2 \mathrm{hr}$., and included a $10 \mathrm{~min}$. rest period midway through.

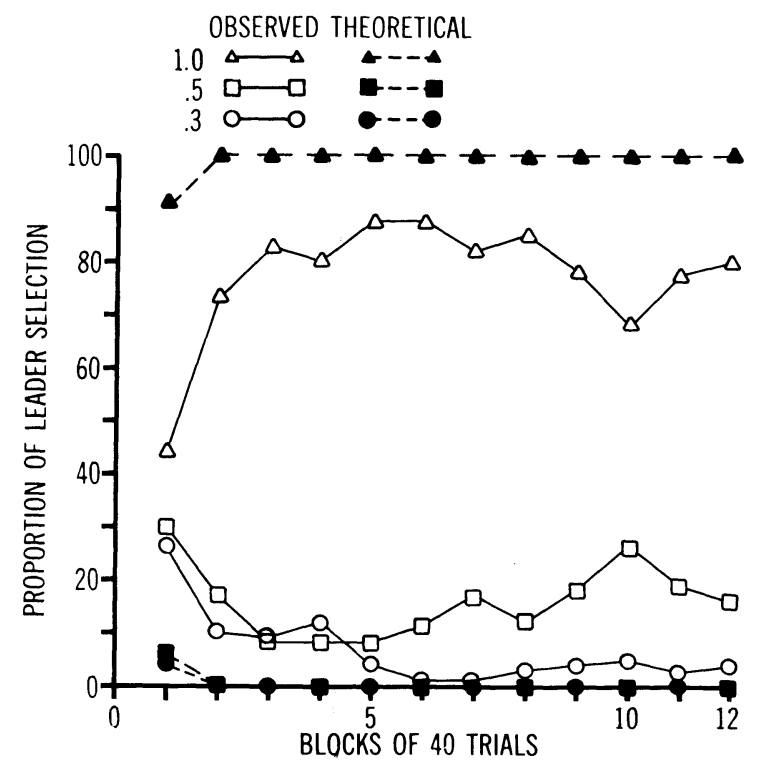

Fig. 1. Learning curves for leader selection. 
TABLE I

Asymptotic Proportions of Leadership and Preference

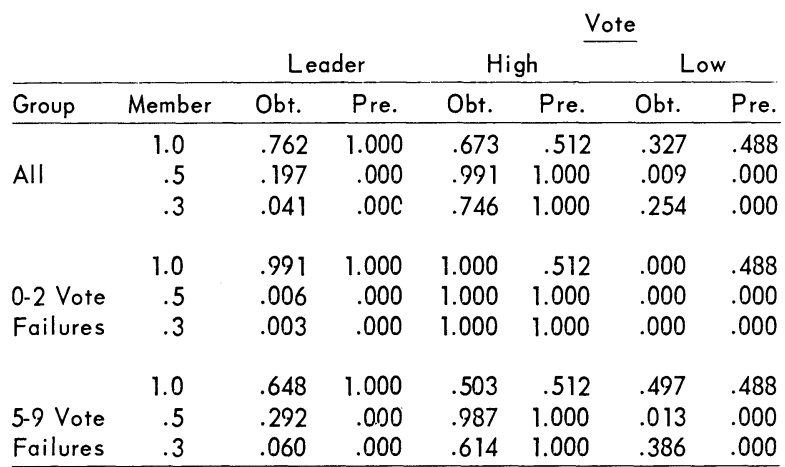

\section{Results and Discussion}

Figure 1 shows the theoretical and obtained learning curves for leader selection in blocks of 40 trials. (The mathematical basis of theoretical prediction may be found in Binder et al, 1965.) Table 1 contains a summary over asymptotic trials (321-480) of obtained proportions each member became leader and also the proportionate voting preferences for all members; the values predicted on the basis of the mathematical model are also shown. It is quite clear that the predictions are in error, because the $\pi=1.0$ member is not chosen consistently.

However, an idiosyncrasy of the experimental method created the predicament that the $\pi=1.0$ member was usually reinforced somewhat less than 100\%. That is, a time interval was allowed for deciding, and an individual who did not vote within the period was considered wrong. The $\pi=1.0$ member failed to vote on only 27 occasions out of 2227 opportunities (slightly over $1 \%$ of the time), but failure did occur more often with some teams than others. If these failures to decide affected voting tendencies, analysis should show their effects predominately in teams which had such failures most frequently. For comparison purposes, the six groups were divided into two sets: one contained a team with 0 voting failure (for the $\pi=1.0$ member) and a team with 2 voting failures; the other set contained teams with 5,6, or 9 voting failures. Table 1 contains a summary over asymptotic trials for these separate groups, similar to the summary discussed above (and presented in the upper part of Table 1) for all Ss. The obtained results for the 0-2 set differ from the predicted by a significant amount only in the voting preferences for the $\pi=1.0$ member. Apparently, when the other members locked onto him as their voting choice he locked onto the $\pi=.5$ member, perhaps to maintain the status quo.

It is interesting that the $\pi=.5$ member provided a very good fit for voting preferences over all groups.

\section{References}

Binder, A., Wolin, B. R., \& Terebinski, S. J. Leadership in small groups: A mathematical approach. J. exp. Psychol., 1965, 69, 126-134.

Binder, A., Wolin, B. R., \& Terebinski, S. J. Leadership in small groups: A resolution of discordance. J. exp. Psychol., 1965a, in press.

Binder, A., Wolin, B. R., \& Terebinski, S. J. Learning and extinction of leadership preferences in small groups. J. exp. Psychol., 1965 b, in press.

\section{Notes}

1. This research was supported by grant GS-507 from the National Science Foundation to the System Development Corporation. 